\title{
LICANOL, UM NOVO FLAVANOL, E OUTROS CONSTITUINTES DE Licania macrophylla Benth
}

\author{
Fernando Antônio de Medeiros e Alessandra A. N. Medeiros \\ Instituto de Pesquisas Científicas e Tecnológicas do Amapá, 68900-260 Macapá - AP, Brasil \\ Josean Fechine Tavares, José Maria Barbosa Filho, Edeltrudes de Oliveira Lima e Marcelo Sobral da Silva* \\ Departamento de Ciências Farmacêuticas, Centro de Ciências da Saúde, Universidade Federal da Paraíba, 58051-900 João Pessoa \\ - PB, Brasil
}

Recebido em 4/10/11; aceito em 6/2/12; publicado na web em 11/4/12

\begin{abstract}
LICANOL, A NEW FLAVANOL, AND OTHER CONSTITUENTS FROM THE Licania macrophylla Benth. The phytochemical investigation of $L$. macrophylla Benth led to the isolation of a new flavanol named licanol: (-)-4'-O-methyl-epigallocatechin-3'-O- $\alpha-$ L-rhamnoside, along with nine known compounds, identified as: (-)-4'-O-methyl-epigallocatechin, pheophytin A, $13^{2}$-hydroxy-(132$S$ )-pheophytin A, pheophytin B, sitosterol, stigmasterol, sitosterol- $\beta$ - $O$-glucoside, betulinic alcohol and oleanolic acid. The structures were established based on IR, HR-ESI-MS, and NMR spectrometric data analysis with the aid of $2 \mathrm{D}$ techniques. The methanolic extracts of leaves and stem bark as well as the compounds licanol, $13^{2}$-hidroxi-(132-S)-feofitina A, and betulinic alcohol demonstrated antimicrobial activity against several bacterial strains.
\end{abstract}

Keywords: Licania macrophylla; Chrysobalanaceae; (-)-4'-O-methyl-epigallocatechin-3'-O- $\alpha$-L-rhamnoside.

\section{INTRODUÇÃO}

A família Chrysobalanaceae é constituída por 18 gêneros e 531 espécies, distribuídas nos dois trópicos. São formadas por árvores ou arbustos. ${ }^{1}$ Espécies dessa família são largamente utilizadas na medicina tradicional, principalmente na África para o tratamento de disenteria, dor de dente, doenças venéreas, epilepsia e malária. ${ }^{2}$ Dentre as principais atividades biológicas comprovadas dessas espécies destaca-se a citotóxica atribuída principalmente aos ácidos betulínico, oleanólico e pomólico. Esses triterpenos pentacíclicos foram isolados das folhas e frutos de Licania tomentosa Benth. e das folhas de Chrysobalanus icaco L., sendo capazes de inibir o crescimento e induzir apoptose de células K562. ${ }^{3}$ Os principais metabólitos secundários de Chrysobalanaceae são os flavonoides, principalmente os glicosilados, ${ }^{4}$ triterpenoides ${ }^{5}$ e diterpenoides. ${ }^{6}$

O gênero Licania Aubl. é constituído por 150 espécies distribuídas amplamente desde o México, Flórida e Antilhas até o sul do Brasil.

Licania macrophylla Benth, conhecida popularmente por "anauera" ou "anuera" é árvore mediana encontrada as margens da várzea das regiões do baixo Amazonas. ${ }^{8}$ Algumas etnias indígenas, ao longo da bacia do Oiapoque, usam a planta como um potente antidisentérico. $\mathrm{Na}$ Guiana Francesa, enquanto os índios Wayãpi utilizam a casca do caule como decocto, os índios Palikur e Creoles preparam um macerado das sementes, sendo ambas as preparações consideradas afrodisíacas pelos caboclos do sul do Pará e Amapá. ${ }^{9}$ No entanto, não há estudos sobre esta espécie.

Nesse trabalho são descritos o isolamento e a determinação estrutural de um novo flavanol (-)-4'-O-metil-epigalocatequina-3'- $O-\alpha-\mathrm{L}$ raminosídeo, licanol (1), juntamente com 9 compostos conhecidos e identificados como (-)-4'-O-metil-epigalocatequina (2), feofitina A (3), $13^{2}$-hidroxi-(132 $\left.-S\right)$-feofitina A (4), feofitina B (5), sitosterol (6), estigmasterol (7), sitosterol- $\beta$ - $O$-glicosídeo (8), álcool betulínico (9) e ácido oleanólico (10), Figura 1. Foi avaliada também a atividade antibacteriana dos extratos metanólicos das folhas, cascas do caule e das substâncias $\mathbf{1}, \mathbf{4}$ e 9.

*e-mail: marcelosobral@ltf.ufpb.br

\section{RESULTADOS E DISCUSSÃO}

A substância (1) foi isolada na forma de um pó vermelho amorfo. O EM-AR-IES mostrou um pico em 467,1575 referente à molécula protonada $[\mathrm{M}+\mathrm{H}]^{+}$, compatível com a fórmula molecular $\mathrm{C}_{22} \mathrm{H}_{27} \mathrm{O}_{11}$ (massa requerida 467,1571).

O espectro de RMN de ${ }^{13} \mathrm{C}$-APT (125 MHz, $\left.\mathrm{CD}_{3} \mathrm{OD}\right)$ de (1) apresentou um total de 22 sinais, sendo 8 atribuídos a átomos de carbono não hidrogenados, 11 a átomos de carbono metínico, 1 a átomo de carbono metilênico, 1 a átomo de carbono metílico e 1 a átomo de carbono metoxílico. A presença de sinais característicos de átomos de carbono em sistemas aromáticos entre $\delta_{\mathrm{C}} 157,97$ e 95,88, juntamente com os sinais de átomos de carbono oximetínico entre $\delta_{\mathrm{C}} 79,65$ e 61,34 sugeriram a estrutura de um flavonoide com uma unidade osídica. Os sinais em $\delta_{\mathrm{C}} 79,65 ; 67,38$ e 29,16 foram atribuídos a C-2, C-3 e C-4, respectivamente, permitindo inferir que (1) corresponde a um flavanol. Os sinais em $\delta_{\mathrm{C}} 101,18 ; 73,82 ; 72,37 ; 72,20 ; 70,97$ e 17,99 confirmam a presença da raminose.

O espectro de RMN de ${ }^{1} \mathrm{H}\left(500 \mathrm{MHz}, \mathrm{CD}_{3} \mathrm{OD}\right)$ de (1) apresentou sinais característicos de hidrogênios ligados a carbonos aromáticos: $\delta_{\mathrm{H}} 6,84(\mathrm{~d}, J=2,0 \mathrm{~Hz}, 1 \mathrm{H}) ; 6,74(\mathrm{dd}, J=2,0$ e $0,5 \mathrm{~Hz}, 1 \mathrm{H}), 5,94$ (d, $J=2,0 \mathrm{~Hz}, 1 \mathrm{H})$ e $5,92(\mathrm{~d}, J=2,0 \mathrm{~Hz}, 1 \mathrm{H})$. Os valores de deslocamentos químicos, bem como as constantes de acoplamentos $(J=2,0$ $\mathrm{Hz}$ ) apresentadas pelos pares de dupletos, foram condizentes com acoplamento meta em anel B e A de flavonoides, respectivamente, e permitiram inferir um padrão 5,7,9,10 tetrassubstituído para o anel A e 1',3',4' e 5'-tetrassubstituído para o anel B. ${ }^{10}$ A constante de acoplamento $J=0,5 \mathrm{~Hz}$ foi atribuída a um acoplamento a longa de distância de H-6' com H-2. Além desses foram observados sinais na região de prótons alifáticos $\left(\delta_{\mathrm{H}} 1,22-5,40\right)$ característicos de hidrogênios oximetínicos, metilênicos, metílico e metoxílico. A presença dos dupletos em $\delta_{\mathrm{H}} 5,40(\mathrm{~d}, J=1,5 \mathrm{~Hz}, 1 \mathrm{H})$ e em $\delta_{\mathrm{H}} 1,22(\mathrm{~d}, J=6,0 \mathrm{~Hz}$, $3 \mathrm{H})$, característicos de próton anomérico (H-1") e metílico (H-6"), respectivamente, confirmou a unidade osídica raminose. Com base no valor da constante de acoplamento do próton anomérico $(J=1,5$ $\mathrm{Hz}$ ), compatível com acoplamento equatorial-equatorial, verificou-se tratar da subunidade $\alpha$-raminose. ${ }^{10-12}$ Foram observados ainda sinais em $\delta_{\mathrm{H}} 4,98(\mathrm{~d}, J=4,5 \mathrm{~Hz}$ ), compatível com $\mathrm{H}$-2, dois duplos dupletos 
<smiles>COc1cc([C@@H]2Oc3cc(O)cc(O)c3C[C@H]2O)cc(O)c1O</smiles>

$1 \mathrm{R}=$

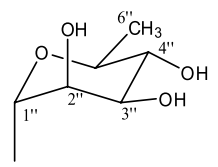

$2 \mathrm{R}=\mathrm{H}$

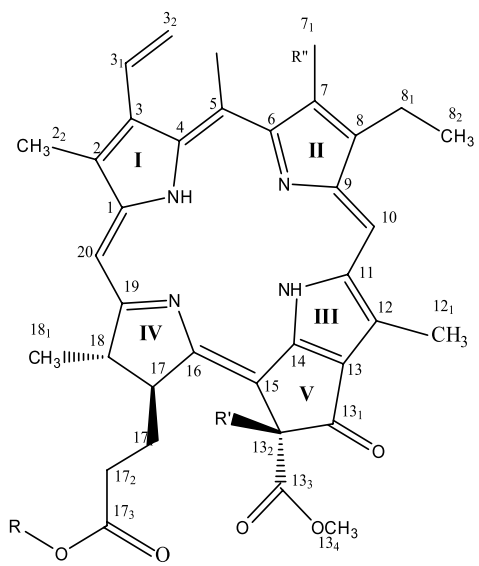

$3 \mathrm{R}=$ Fitil; $\mathrm{R}^{\prime}=\mathrm{H} ; \mathrm{R}^{\prime \prime}=\mathrm{CH}_{3}$

$4 \mathrm{R}=$ Fitil; $\mathrm{R}^{\prime}=\mathrm{H} ; \mathrm{R}^{\prime \prime}=\mathrm{COH}$

$5 \mathrm{R}=$ Fitil; $\mathrm{R}^{\prime}=\mathrm{OH} ; \mathrm{R}^{\prime \prime}=\mathrm{CH}_{3}$<smiles>CC(=CCI)CCCC(C)CCCC(C)CCCC(C)C</smiles>

Fitil

$6 \mathrm{R}=\mathrm{H}$

$7 \mathrm{R}=\mathrm{H}, \Delta^{22-23}$

$8 \mathrm{R}=$ Glicose

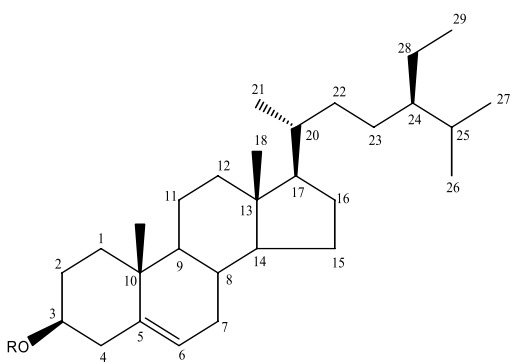

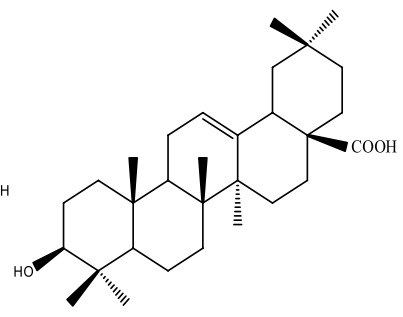

10

em $\delta_{\mathrm{H}} 2,87$ (dd, $J=16,5$ e $\left.3,0 \mathrm{~Hz}, 1 \mathrm{H}\right)$ e em $\delta 2,71$ (dd, $J=16,5$ e $4,5 \mathrm{~Hz}, 1 \mathrm{H})$, característicos dos átomos de hidrogênios metilênicos-benzílico (H-4) de esqueleto flavanol.

Através da análise do espectro de correlação ${ }^{1} \mathrm{H} \mathrm{x}{ }^{13} \mathrm{C}$-HMQC (500 $\mathrm{MHz}, \mathrm{CD}_{3} \mathrm{OD}$ ) de (1) foi possível assinalar os carbonos oximetínicos, metínicos, metilênico, metoxílico e metílico. A correlação observada no espectro ${ }^{1} \mathrm{H} \mathrm{x}{ }^{13} \mathrm{C}-\mathrm{HMBC}$ entre o sinal em $\delta_{\mathrm{H}} 4,82(\mathrm{H}-2)$ com o sinal em $\delta_{\mathrm{C}} 157,05$ confirmou a atribuição desse sinal para o carbono C-9, assim como a correlação de C-9 com o sinal em $\delta_{\mathrm{H}} 5,92$ permitiu identificar H-8. A correlação de H-4 $\left(\delta_{\mathrm{H}} 2,71-2,86\right)$ com o sinal em $\delta_{\mathrm{C}} 157,97$ permitiu atribuir esse sinal ao carbono C-5. Também foi observada a correlação de C-5 $\left(\delta_{\mathrm{C}} 157,97\right)$ com o sinal em $\delta_{\mathrm{H}} 5,94$ confirmando H-6, que, devido a sua correlação direta (HMQC) com o sinal em $\delta_{\mathrm{C}} 96,53$, confirmou a atribuição de C-6. Observaram-se, ainda, correlações entre o sinal em $\delta_{\mathrm{H}} 4,82(\mathrm{H}-2)$ com os sinais em $\delta_{\mathrm{C}}$ 136,$71 ; 108,48$ e 110,41, que confirmaram a atribuição a C-1'e os dois últimos para C-2' e C- 6'. As correlações observadas entre $\delta_{\mathrm{H}} 6,74 \mathrm{e}$ 6,84 (H-6' e H-2') com o sinal em $\delta_{\mathrm{C}} 138,55$ confirmaram esse sinal para o carbono C-4'. Uma correlação intensa observada entre $\delta_{\mathrm{H}} 3,81$ $\left(-\mathrm{OCH}_{3}\right)$ com $\delta_{\mathrm{C}} 138,55$ indicou que o grupo metoxílico se encontra ligado ao carbono C-4'. Também foi observada uma correlação entre o sinal do hidrogênio anomérico em $\delta_{\mathrm{H}} 5,39$ (H-1") com o sinal em $\delta_{\mathrm{C}}$ 151,04 , permitindo identificar o carbono ao qual a raminose estava ligada. Os demais dados estão compilados na Tabela 1.

A estereoquímica de (1) foi proposta com base na constante de acoplamento de $\mathrm{H}-2(J=4,5 \mathrm{~Hz})$, cujo valor remete a um acoplamento equatorial-equatorial, sugerindo os substituintes de C-2 e C-3 em posições pseudoaxiais (Cis). $\mathrm{O}$ valor do $[\alpha]_{\mathrm{D}}^{25}-7,0\left(\right.$ c. $\left.0,001, \mathrm{CH}_{3} \mathrm{OH}\right)$ confirma (1) como sendo um derivado do enantiômero (-)-epicatequina. O espectro de correlação ${ }^{1} \mathrm{H} \times{ }^{1} \mathrm{H}-\mathrm{NOESY}$ mostrou uma correlação entre o sinal do hidrogênio anomérico $\mathrm{H}-1$ ” $\left(\delta_{\mathrm{H}} 5,39\right)$ com o sinal do hidrogênio H-2' $\left(\delta_{\mathrm{H}} 6,84\right)$; foi observada ainda a correlação dos hidrogênios H-6" $\left(\delta_{\mathrm{H}} 1,24\right)$ com o hidogênio metoxílico $\left(\delta_{\mathrm{H}} 3,81\right)$, corroborando com a posição da ramnose em C-3'. Portanto, com base nos dados apresentados foi possível elucidar a estrutura química, bem como propor a estereoquímica de (1), que foi denominada de (-)-4'-O-metilepigalocatequina-3'-O- $\alpha$-L-raminosídeo, substância não descrita ainda na literatura à qual foi atribuído o nome trivial de licanol.

As substâncias 2-10 foram identificadas com base em análises de dados espectroscópicos e comparação com a literatura: (-)-4'-O-metilepigalocatequina, ${ }^{13}$ feofitina $\mathrm{A},{ }^{14} 13^{2}$-hidroxi- $\left(13^{2}-S\right)$-feofitina $\mathrm{A},{ }^{15}$ feofitina $\mathrm{B},{ }^{16}$ sitosterol, ${ }^{17}$ estigmasterol, ${ }^{17}$ sitosterol- $\beta$ - $O$-glicosídeo, ${ }^{18}$ álcool betulínico, ${ }^{17}$ ácido oleanólico, ${ }^{19}$ respectivamente. Segundo Silva e colaboradores, ${ }^{20}$ é possível que feofitinas ocorram naturalmente em vegetais; essa evidencia foi detectada pela observação de pigmentos escuros nas folhas do vegetal in natura, no entanto, a hipótese desses pigmentos serem produtos de degradação da clorofila não está inteiramente descartada.

O extrato bruto de L. macrophylla demonstrou atividade antibacteriana contra bactéria multirresistente a drogas. ${ }^{21}$ Nesse trabalho, o ensaio da concentração inibitória mínima (CIM) revelou que o extrato metanólico do caule (EMC) de L. macrophylla foi ativo contra as linhagens de Staphylococcus aureus ATCC 25928, Pseudomonas aeruginosa ATCC 25853 e Escherichia coli ATCC 10536. O EMC apresentou atividade antibacteriana moderada para a linhagem de Staphylococcus aureus ATCC 25928 e fraca para Pseudomonas aeruginosa ATCC 25853 e Escherichia coli ATCC 10536, enquanto (1) apresentou atividade relevante para Staphylococcus aureus ATCC 25928 e Pseudomonas aeruginosa ATCC 25853 e fraca atividade para Escherichia coli ATCC 10536. Este resultado sugeriu que a atividade antibacteriana do EMC provavelmente se deve em parte à presença de (1) nesse extrato. O EMF foi considerado moderadamente ativo contra

Figura 1. Constituintes químicos isolados de L. macrophylla 
Tabela 1. Dados de $\mathrm{RMN}{ }^{1} \mathrm{H}$ e de ${ }^{13} \mathrm{C}$ uni e bidimensional de $\mathbf{1}$

\begin{tabular}{|c|c|c|c|c|c|}
\hline \multirow{2}{*}{ Posição } & \multicolumn{2}{|c|}{${ }^{1} \mathrm{H} x{ }^{13} \mathrm{C}-\mathrm{HMQC}$} & \multicolumn{2}{|c|}{${ }^{1} \mathrm{H} \mathrm{x}{ }^{13} \mathrm{C}-\mathrm{HMBC}$} & \multirow{2}{*}{${ }^{1} \mathrm{H} x{ }^{1} \mathrm{H}-\mathrm{COSY}$} \\
\hline & $\delta_{\mathrm{H}}$ & $\delta_{\mathrm{C}}$ & ${ }^{2} J_{\mathrm{CH}}$ & ${ }^{3} J_{\mathrm{CH}}$ & \\
\hline 2 & $4,82(1 \mathrm{H}, \mathrm{d}, J=4,5)$ & 79,65 & $\mathrm{C}-1$ & C-2'; C-6'; C-4 & $\mathrm{H}-3$ \\
\hline 3 & $4.20(1 \mathrm{H}, \mathrm{m})$ & 67,38 & & & H-2 \\
\hline $4 \mathrm{ax}$ & $2,86(1 \mathrm{H}, \mathrm{dd}, J=4.5 ; 16.5)$ & 29,04 & C-10 & $\mathrm{C}-5 ; \mathrm{C}-9$ & $\mathrm{H}-4 \mathrm{eq} ; \mathrm{H}-3$ \\
\hline $4 \mathrm{eq}$ & $2,71(1 \mathrm{H}, \mathrm{dd}, J=3,0 ; 16,5)$ & & C-10 & $\mathrm{C}-5 ; \mathrm{C}-9 ; \mathrm{C}-2 ; \mathrm{C}-3$ & H-4ax; H-3 \\
\hline 5 & & 157,97 & & & \\
\hline 6 & $5,94(1 \mathrm{H}, \mathrm{d}, J=2,0)$ & 96,53 & C-5 & C-10; C-8 & \\
\hline 7 & & 157,71 & & & \\
\hline 8 & $5,92(1 \mathrm{H}, \mathrm{d}, J=2,0)$ & 95,88 & C-9 & C-10; C-6 & \\
\hline 9 & & 157,05 & & & \\
\hline 10 & & 100,07 & & & \\
\hline 1 ' & & 136,71 & & & \\
\hline $2^{\prime}$ & $6,84(1 \mathrm{H}, \mathrm{d}, J=2,0)$ & 108,48 & C-3' & C-6'; C-4' & \\
\hline $3^{\prime}$ & & 151,04 & & & \\
\hline $4^{\prime}$ & & 138,55 & & & \\
\hline 5 & & 151,59 & & & \\
\hline $6^{\prime}$ & $6,74(1 \mathrm{H}, \mathrm{d}, J=2,0 ; 0,5)$ & 110,41 & C-5 & C-2'; C-4' & \\
\hline $1 "$ & $5,39(1 \mathrm{H}, \mathrm{d}, J=1,5)$ & 101,18 & & $\mathrm{C}-3{ }^{\prime} ; \mathrm{C}-5^{\prime \prime} ; \mathrm{C}-3{ }^{\prime \prime}$ & $\mathrm{H}-2 ”$ \\
\hline $2 "$ & $4,06(1 \mathrm{H}, \mathrm{dd}, J=3,5 ; 1,5)$ & 72,20 & $\mathrm{C}-3 "$ & C-4” & $\mathrm{H}-1 ”$ \\
\hline $3 "$ & $3,88(1 \mathrm{H}, \mathrm{dd}, J=3,5 ; 10,0)$ & 72,37 & $\mathrm{C}-4 "$ & & $\mathrm{H}-4 ”$ \\
\hline $4 "$ & $3,46(1 \mathrm{H}, \mathrm{dd}, J=10,0 ; 10.0)$ & 73,82 & $\mathrm{C}-3 ", \mathrm{C}-5 "$ & & H-5"; H-3" \\
\hline $5 "$ & $3,79(1 \mathrm{H}, \mathrm{m})$ & 70,97 & & & H-6"; H-4" \\
\hline $6 "$ & $1,24(3 \mathrm{H}, \mathrm{d}, J=6,0)$ & 17,99 & $\mathrm{C}-5 "$ & C-4" & $\mathrm{H}-5$, \\
\hline $\mathrm{OCH}_{3}$ & $3,81(3 \mathrm{H}, \mathrm{s})$ & 61,34 & & C-4' & \\
\hline
\end{tabular}

as linhagens de Staphylococcus aureus ATCC 25923, Escherichia coli ATCC10536 e Salmonella enterica ATCC 6017 e para as demais cepas foi considerado de atividade antibacteriana fraca, Tabela 2.

Já (9) foi ativo contra a maioria das cepas bacterianas testadas, apresentando atividade relevante para a linhagem de Staphylococcus aureus ATCC 25923 e fraca atividade para as linhagens de Staphylococcus aureus ATCC 13150, Staphylococcus epidermides
ATCC 12228, Escherichia coli ATCC 11105, Escherichia coli ATCC 18739, Escherichia coli ATCC 10536 e Shigella flexiner MM 412.

A substância (4) apresentou atividade significante para as linhagens de Staphylococcus aureus ATCC 13150, Staphylococcus aureus ATCC 25923, Pseudomonas aeruginosa ATCC 25853 e Escherichia coli ATCC 10536 e fraca atividade para todas as demais cepas testadas, Tabela 2. Comparando as atividades de EMF, (4) e

Tabela 2. Concentração inibitória mínima do EMC e EMF de L. macrophylla e compostos 1, 4 e 9

\begin{tabular}{|c|c|c|c|c|c|c|c|}
\hline Microrganismos & $\begin{array}{c}\text { EMF } \\
(\mu \mathrm{g} / \mathrm{mL})\end{array}$ & $\begin{array}{c}\text { EMC } \\
(\mu \mathrm{g} / \mathrm{mL})\end{array}$ & $\begin{array}{c}1 \\
(\mu \mathrm{g} / \mathrm{mL})\end{array}$ & $\begin{array}{c}\mathbf{4} \\
(\mu \mathrm{g} / \mathrm{mL})\end{array}$ & $\begin{array}{c}9 \\
(\mu \mathrm{g} / \mathrm{mL})\end{array}$ & $\begin{array}{c}\text { cloranfenicol } \\
(50 \mu \mathrm{g} / \mathrm{mL})\end{array}$ & crescimento \\
\hline S. aureus ATCC 13150 & 1000 & ND & ND & 50 & 100 & - & + \\
\hline S. aureus ATCC 25923 & 500 & 250 & 25 & 25 & 50 & - & + \\
\hline S. epidermidis ATCC 12228 & 1000 & ND & ND & 100 & 100 & - & + \\
\hline P. aeruginosa ATCC 25853 & 1000 & 1000 & 50 & 50 & 100 & - & + \\
\hline E. coli ATCC 11105 & 1000 & ND & ND & 100 & 100 & - & + \\
\hline E. coli ATCC 18739 & 1000 & ND & ND & 100 & 100 & - & + \\
\hline E. coli Clássica & 1000 & ND & ND & 100 & ND & - & + \\
\hline E. coli ATCC 10536 & 500 & 1000 & 100 & 50 & 100 & - & + \\
\hline S. flexiner MM 412 & 1000 & ND & ND & 100 & 100 & - & + \\
\hline S. sonnei LM 07 & 1000 & ND & ND & 100 & ND & - & + \\
\hline Salmonella spp. LM 08 & 1000 & ND & ND & 100 & ND & - & + \\
\hline S. enterica ATCC 6017 & 500 & ND & ND & 100 & ND & - & + \\
\hline
\end{tabular}

(+): crescimento do microrganismo no meio de cultura; (-): ausência do crescimento do microrganismo no meio de cultura; (ND): não detectado 
(9), percebeu-se uma semelhança entre as atividades do extrato e dos compostos.

\section{PARTE EXPERIMENTAL}

\section{Procedimentos experimentais gerais}

Os espectros de RMN foram obtidos em espectrômetro MercuryVarian a $200 \mathrm{MHz}{ }^{1} \mathrm{H}$ e $50 \mathrm{MHz}{ }^{13} \mathrm{C}$ e Varian Systems a $500 \mathrm{MHz}$ ${ }^{1} \mathrm{H}$ e $125 \mathrm{MHz}{ }^{13} \mathrm{C}$. Os solventes empregados foram $\mathrm{CD}_{3} \mathrm{OD}, \mathrm{CDCl}_{3}$ e $\mathrm{C}_{5} \mathrm{D}_{5} \mathrm{~N}$, cujos sinais característicos nos espectros de $\mathrm{RMN}{ }^{1} \mathrm{H}$ e de ${ }^{13} \mathrm{C}$ serviram para ajuste de escala de frequência. O espectro de massas de alta resolução foi obtido em espectrômetro Brüker Daltonics MicfroTOF-II operando no modo IES positivo. A rotação óptica foi obtida à temperatura ambiente, em polarímetro Jasco modelo P-2000. Para as cromatografias em coluna utilizou-se como fase estacionária sílica gel 60 (70-230 Mesh) da Merck. A cromatografia em camada delgada comparativa (CCDC) foi empregada para análise e reunião das frações obtidas por cromatografia em coluna. Foram utilizadas placas de vidro cujas fases fixas foram preparadas com suspensão de sílica gel PF254 7749 (Merck) em água. As substâncias em análise foram evidenciadas pelo uso de radiação ultravioleta nos comprimentos de onda de 254 e $366 \mathrm{~nm}$ e pela impregnação das placas em cubas de vidro saturadas por vapores de iodo.

\section{Material vegetal}

As cascas do caule e folhas de L. macrophylla Benth (Chrysobalanaceae) foram coletadas em outubro de 2005, na Reserva Florestal do Instituto de Pesquisas Científicas e Tecnológicas do Estado do Amapá (IEPA), localizada no município de Porto Grande, Amapá, Brasil. Uma exsicata encontra-se depositada no Herbário Amapaense HAMAB do IEPA sob o número 011310.

\section{Extração e isolamento}

As cascas do caule $(2,3 \mathrm{~kg})$ e folhas $(600 \mathrm{~g})$ secas e trituradas de L. macrophylla foram submetidas à extração por maceração com metanol comercial, à temperatura ambiente, por 10 dias. As soluções extrativas obtidas foram concentradas em evaporador rotativo sob temperatura controlada $\left(45^{\circ} \mathrm{C}\right)$, obtendo-se o extrato metanólico das cascas do caule (EMC) e o extrato metanólico das folhas (EMF), com rendimentos de $\mathrm{EMC}=15 \%$ (345 g) e $\mathrm{EMF}=7 \%$ (42 g).

$\mathrm{O}$ EMC de L. macrophylla $(20 \mathrm{~g})$ foi submetido à cromatografia em coluna usando-se como adsorvente $500 \mathrm{~g}$ de sílica gel 60 da Merck (Art. 7734) empacotada em suporte de vidro, procedendo-se à eluição com hexano, acetato de etila e metanol, puros ou em mistura binárias, em ordem crescente de polaridade, obtendo-se 150 frações de $100 \mathrm{~mL}$ cada. A fração 6 que foi eluída num sistema de solvente hexano:acetato de etila (8:2) foi recristalizada com clorofórmio, obtendo-se 2 (15 mg). As frações Fr-9-17 foram filtradas em sílica gel com gradiente de polaridade acetato de etila e metanol, obtendo-se 30 frações de $50 \mathrm{~mL}$ cada. A fração eluída com acetato de etila:metanol $3 \%$ foi recristalizada com acetona obtendo-se $\mathbf{1}$ (30 mg).

$\mathrm{O}$ EMF de L. macrophylla Benth (10 g) foi cromatografado em coluna usando-se como adsorvente $300 \mathrm{~g}$ de sílica gel 60 da Merck (Art. 7734) e como eluentes hexano, clorofórmio, acetato de etila e metanol, puros ou em mistura binária, em gradiente crescente de polaridade. Foram coletadas frações de $120 \mathrm{~mL}$, num total de 254, as quais foram monitoradas por cromatografia em camada delgada analítica (CCDA) e reunidas de acordo com a semelhança de seus Rf. As frações 62 a 66 eluídas em hexano:acetato de etila (8:2) apresentaram-se como cristais incolores, que, após analisadas por CCDA, revelaram duas bandas características de uma mistura de duas substâncias 6 e 7 (25 mg). A fração 87-96 eluída em hexano:acetato de etila (1:1) foi submetida à cromatografia em camada delgada preparativa com hexano e acetato de etila (1:1), eluída 3 vezes, obtendo-se 3 (21 mg) e 4 (12 mg). A fração 97 eluída em hexano:acetato de etila (3:7) foi recristalizada em metanol, obtendo-se 8 (40 mg). A fração 111 foi filtrada em sílica gel com gradiente de hexano:acetato de etila, obtendo-se 5 (30 mg) que foi eluída na proporção (7:3). A fração 113 foi recristalizada em metanol obtendo-se $\mathbf{9}(50 \mathrm{mg})$. As frações 178 a 181 formaram um precipitado em forma de pó branco, insolúvel em metanol. Estas foram purificadas através de sucessivas lavagens com metanol, obtendo-se $\mathbf{1 0}$ (60 mg).

\section{Ensaios microbiológicos}

Os extratos e substâncias isoladas de L. macrophylla Benth foram avaliadas quanto a sua capacidade de inibir o crescimento in vitro de bactérias patogênicas. Para a realização do teste da CIM foi utilizado o método de microdiluição do caldo nutriente, empregando 12 cepas bacterianas, sendo 3 Gram-positivas (Staphylococcus aureus ATCC 13150 , Staphylococcus aureus ATCC 25923 e Staphylococcus epidermidis ATCC 25923) e 6 Gram-negativas (Pseudomonas aeruginosa ATCC 25853, Escherichia coli ATCC 11105, Escherichia coli ATCC 18739, Escherichia coli Clássica, Escherichia coli ATCC 10536, Shigella flexiner MM 412, Shigella sonnei LM 07, Salmonella spp LM 08 e Salmonella enterica ATCC 6017). Foram feitos para o crescimento de cada microrganismo, como também com antibacteriano cloranfenicol (50 $\mu \mathrm{g} / \mathrm{mL})$. Para classificação da atividade antimicrobiana foram considerados os seguintes critérios: atividade entre 10 e $100 \mu \mathrm{g} / \mathrm{mL}$ foi considerada como relevante; 101 a $500 \mu \mathrm{g} / \mathrm{mL}$, como moderada; 501 a $1000 \mu \mathrm{g} / \mathrm{mL}$, como fraca e acima de $1000 \mu \mathrm{g} / \mathrm{mL}$ como inativa. ${ }^{22}$

(-)-4'-O-metil-epigalocatequina-3'-O- $\alpha$-L-raminosídeo, licanol (1) Infravermelho em KBr $v_{\max } / \mathrm{cm}^{-1}: 3450,1651,1500,1357 ;[\alpha]_{\mathrm{D}}^{25}$ -7,0 (c. 0,001, $\left.\mathrm{CH}_{3} \mathrm{OH}\right)$, EM-AR-IES m/z: 467,1575 (M+H) ${ }^{+}$, (calculado para $\left.\mathrm{C}_{22} \mathrm{H}_{27} \mathrm{O}_{11} 467,1574\right),{ }^{1} \mathrm{H} \mathrm{e} \mathrm{e}^{13} \mathrm{C}$, Tabela 1 .

\section{MATERIAL SUPLEMENTAR}

Espectros de RMN 1D e 2D de licanol estão disponíveis em http:// quimicanova.sbq.org.br, na forma de arquivo PDF e com acesso livre.

\section{AGRADECIMENTOS}

Ao CNPq, CAPES e FAPESQ-PB pelo apoio financeiro e ao LMCA-Central Analítica da UFPB pela obtenção dos espectros.

\section{REFERÊNCIAS}

1. Prance, G. T.; Rodriguésia 2007, 58, 493.

2. Lee, K. S.; Shamon, L. A.; Chai, H. B.; Chagwedera, T. E.; Besterman, J. M.; Farnsworth, N.; Cordell, G. A.; Pezzuto, J. M.; Kinghorn, D.; Chem.-Biol. Interact. 1996, 99, 193.

3. Fernandes, J.; Castilho, R. O.; Costa, M. R.; Wagner-Souza, K.; Kaplan, M. A. C.; Gattass, C. R.; Cancer Lett. 2003, 190,165.

4. Braca, A.; Luna, D.; Mendez, J.; Biochem. Syst. Ecol. 2002, 30, 271; Braca, A.; Tommasi, N. D.; Mendez, J.; Morelli, I.; Biochem. Syst. Ecol. 1999, 27, 527.

5. Braca, A.; Sortino, C.; Mendez, J.; Morelli, I.; Fitoterapia 2001, 72, 585; Castilho, R. O.; Oliveira, R. R.; Kaplan, M. A. C.; Fitoterapia 2005, 76, 562.

6. Chaudhuri, S. K.; Badisa, R. B.; Pilarinou, E.; Walker, E.; Nat. Prod. Lett. 2002, 16, 39. 
7. Andrade, E. H. A.; Zoghbi, M. G. B.; Maia, J. G. S.; Acta Amazon. 1998 , 28,55 .

8. Rodrigues, R. M.; Belém: CEJUP 1989, 33.

9. Grenand, P.; Moretti, C.; Jacquemin, H.; Pharmacopées Traditionnelles en Guyane, Orstom: Paris, 1987.

10. Regasini, L. O; Fernandes, D. C.; Castro-Gamboa, I.; Silva, D. H. S.; Furlan, M.; Bolzani, V. S.; Barreiro, E. J.; Cardoso-Lopes, E. M.; Young, M. C. M.; Torres, L. B.; Vellosa, J. C. R.; Oliveira, O. M. M.; Quim. Nova 2008, 31, 802 .

11. Ohmori, K.; Ohrui, H.; Suzuki, K.; Tetrahedron Lett. 2000, 41, 5537.

12. Hussein, G.; Nakamura, N.; Meselhy, R. M.; Hattori, M.; Phytochemistry 1999, 50, 689.

13. Agrawal, P. K.; Carbon-13 NMR of Flavonoids, Elsevier: Amsterdan, 1989.

14. Melos, J. L. R.; Honda, N. K.; Quim. Nova 2007, 30, 292.

15. Tomaz, A. C. A.; Nogueira, R. B. S. S.; Pinto, D. S.; Agra, M. F.; Souza, M. F. V.; Cunha, E. V. L.; Rev. Bras. Farmacogn. 2008, 18, 47.
16. Schwikkard, S. L.; Mulholland, D. A.; Hutchings, A.; Phytochemistry 1998, 49, 2391.

17. Falcão, D. Q.; Dissertação de Mestrado, Universidade Federal do Rio de Janeiro, Brasil, 2003.

18. Kojima, H.; Sato, N.; Hatano, A.; Ogura, H.; Phytochemistry 1990, 29 , 2351.

19. Mahato, S. B.; Sen, S.; Phytochemistry 1997, 44, 1185.

20. Silva, T. M. S.; Câmara, C. A.; Barbosa-Filho, J. M.; Giulietti, A. M.; Quim. Nova 2010, 33, 571 .

21. Correia, A. F.; Segovia, J. F. O.; Gonçalves, M. C. A.; Oliveira, V. L.; Silveira, D.; Carvalho, J. C. T.; Kanzaki, L. I. B.; Eur.Rev. Med. Pharmacol. Sci. 2008, 12, 369; Petto, J. B.; J. Ethnopharmacol. 2004, 90, 135.

22. Holetz, F. B.; Pessini, G. L.; Sanches, N. R.; Mem. Inst. Oswaldo Cruz. 2002, 97, 1027. 